\title{
Research Article Delay-Dependent Stability Criteria for Singular
Systems with Interval Time-Varying Delay
}

\author{
Jianmin Jiao \\ Department of Mathematics, Baoji University of Arts and Sciences, Baoji 721013, China \\ Correspondence should be addressed to Jianmin Jiao, jmjiao@126.com
}

Received 8 September 2012; Revised 15 November 2012; Accepted 19 November 2012

Academic Editor: Zheng-Guang Wu

Copyright (C) 2012 Jianmin Jiao. This is an open access article distributed under the Creative Commons Attribution License, which permits unrestricted use, distribution, and reproduction in any medium, provided the original work is properly cited.

This paper is concerned with stability analysis for singular systems with interval time-varying delay. By constructing a novel Lyapunov functional combined with reciprocally convex approach and linear matrix inequality (LMI) technique, improved delay-dependent stability criteria for the considered systems to be regular, impulse free, and stable are established. The developed results have advantages over some previous ones as they involve fewer decision variables yet less conservatism. Numerical examples are provided to demonstrate the effectiveness of the proposed stability results.

\section{Introduction}

It is well known that time delays frequently occur in many practical systems, such as biological systems, chemical systems, electronic systems, and network control systems. The time delays are regarded as the major source of oscillation, instability, and poor performance of dynamic systems. During the last two decades, there has been some remarkable theoretical and practical progress in stability, stabilization, and robust control of linear time-delay systems $[1,2]$. Currently, the results of stability for time-delay systems mainly focus on timevarying delay with range zero to an upper bound. However, in practice, the delay range may have a nonzero lower bound, and such systems are referred to interval time-varying delay systems. Typical examples for interval time-delay systems are networked control systems [3]. With rapid advancement in the networked control systems technology, a number of significant results have been reported in the recent past for the stability of interval time-delay systems [3-14]. For example, in [3], a discretized Lyapunov functional approach is employed to obtain stability criteria for linear uncertain systems with interval time-varying delays. By using free-weighting matrices, $[4,5]$ present some less conservative stability conditions. The free-weighting matrices method was further improved in $[6,7]$ by constructing augmented 
Lyapunov functionals. The free-weighting matrices method is regarded as an effective way to reduce the conservatism of the stability results; however, one chief shortcoming is that too many free-weighting matrices introduced in the theoretical derivation sometimes cannot reduce the conservatism of the obtained results, on the contrary, they make criteria mathematically complex and computationally less effective. In [8,9], via different Lyapunov functionals with fewer matrix variables whose derivative is estimated using Jensen inequality, some simple stability criteria were obtained, these results were improved in [10] using the convex analysis method, and the result in [10] was further improved in [11] using the reciprocally convex approach. Recently, by introducing some integral terms in the augmented vector and using the Lyapunov functionals with triple-integral terms, some less conservative results were obtained in [12-14].

Singular systems, which are also referred to as descriptor systems, differential algebraic systems, or semistate systems whose behaviors are described by differential equations (or difference equations) and algebraic equations. Singular systems have strong practical relevance in a variety of physical processes such as power systems, social economic systems, and circuit systems [15]. For this reason, singular systems have attracted a lot of researches from mathematics and control communities. A great number of fundamental results based on the theory of regular systems have been extended to the area of singular systems [16]. Recently, more and more attention has been paid to singular systems with delay. Singular time-delay systems can preserve the structure of practical systems and have extensive applications in various engineering systems, including aircraft attitude control, flexible arm control of robots, large-scale electric network control, chemical engineering systems, and lossless transmission lines [17]. It is well known that the stability analysis for singular systems is much more complicated than that for regular systems because it requires to consider not only stability, but also regularity and absence of impulse (for continuous singular systems) [18-28] or causality (for discrete singular systems) [29-32]. In order to obtain stability conditions of singular time-delay systems, many efforts have been made in the literature, among which the model transformation and bounding technique for cross-terms are often used [18-20]. However, it is well known that these two kinds of methods are the main source of conservatism. Without using model transformation and bounding technique for cross-terms, some improved stability conditions with less conservatism have been provided by introducing free-weighting matrices [21,22], integral inequality [23, 24], delay decomposition [25], and parameterized Lyapunov functional [26]. However, the involved time delays of [18-26] are all time invariant, which limits the scope of applications of the given results. In the case where time-varying delays appear in singular systems, some stability results were proposed in $[27,28]$. The range of time-varying delay considered in $[27,28]$ is from zero to an upper bound. In the case of the lower bound of delay is not restricted to be zero, the stability criteria in $[27,28]$ are conservative because they do not take into account the information of the lower bound of delay. Very recently, singular systems with time-varying delay in a range are studied in [33-38]. Nevertheless, there still exists some room for deriving less conservative as well as computationally less expensive stability criteria, which has motivated this paper.

In this paper, we will construct a novel Lyapunov functional and extend the reciprocally convex approach inspired by Park et al. [11] to analyze the stability of singular systems with interval time-varying delay. Some improved results for the considered systems to be regular, impulse free, and stable are established in terms of LMIs. The obtained stability criteria involve fewer decision variables comparable to those based on the free-weighting matrices method; hence they are mathematically less complex and computationally more efficient. 
Meanwhile, the new criteria are less conservative than existing ones, which will be demonstrated by some numerical examples.

Notations. Throughout this paper, $\mathbf{R}^{n}$ denotes the $n$-dimensional Euclidean space, while $\mathbf{R}^{m \times n}$ refers to the set of all real matrices with $m$ rows and $n$ columns. $A^{T}$ represents the transpose of the matrix $A$, while $A^{-1}$ denotes the inverse of $A$. For real symmetric matrices $X$ and $Y$, the notation $X \geq Y$ (resp., $X>Y$ ) means matrix $X-Y$ is positive semidefinite (resp., positive-definite). $I$ is the identity matrix with appropriate dimensions. $\|x\|$ refers to the Euclidean norm of the vector $x$, that is, $\|x\|=\sqrt{x^{T} x}$.

\section{Problem Formulation and Preliminaries}

Consider the singular system with interval time-varying delay described by:

$$
\begin{gathered}
E \dot{x}(t)=A x(t)+B x(t-d(t)), \\
x(\theta)=\varphi(\theta), \quad \theta \in\left[-d_{2}, 0\right],
\end{gathered}
$$

where $x(t) \in \mathbf{R}^{n}$ is the state vector, and $\varphi(\theta) \in \mathbf{R}^{n}$ is a continuous vector-valued initial function of $\theta \in\left[-d_{2}, 0\right]$. The matrix $E \in \mathbf{R}^{n \times n}$ may be singular, and it is assumed that rank $E=$ $r \leq n, A, B \in \mathbf{R}^{n \times n}$ are known real constant matrices with appropriate dimensions. $d(t)$ is the time-varying delay and is assumed to satisfy

$$
d_{1} \leq d(t) \leq d_{2}, \quad \dot{d}(t) \leq \mu,
$$

where $0<d_{1}<d_{2}$ and $0 \leq \mu<1$ are known constants; $d_{1}$ and $d_{2}$ represent the lower and upper bounds of the time-varying $d(t)$, respectively, $\mu$ is the bound on the delay derivative.

The purpose of this paper is to formulate new delay-dependent criteria to check the stability of singular time-delay system (2.1). Let us give the following definitions and lemmas, which will play an indispensable role in deriving our criteria.

Definition 2.1 (see [16]). (i) The pair $(E, A)$ is said to be regular if $\operatorname{det}(s E-A)$ is not identically zero. (ii) The pair $(E, A)$ is said to be impulse free if $\operatorname{deg}(\operatorname{det}(s E-A))=\operatorname{rank} E$.

Definition 2.2 (see [35]). (i) The singular time-delay system (2.1) is said to be regular and impulse free if the pairs $(E, A)$ and $(E, A+B)$ are regular and impulse free. (ii) The singular time-delay system (2.1) is said to be stable if for any $\varepsilon>0$, there exists a scalar $\delta(\varepsilon)>0$ such that, for any compatible initial conditions $\varphi(t)$ satisfying $\sup _{-d_{2} \leq t \leq 0}\|\varphi(t)\| \leq \delta(\varepsilon)$, the solution $x(t)$ of system (2.1) satisfies $\|x(t)\| \leq \varepsilon$ for any $t \geq 0$, more over $\lim _{t \rightarrow \infty} x(t)=0$.

Definition 2.3 (see [11]). Let $\phi_{1}, \phi_{2}, \ldots, \phi_{N}: \mathbf{R}^{m} \mapsto \mathbf{R}^{n}$ be a given finite number of functions such that they have positive values in an open subset $\mathbf{D}$ of $\mathbf{R}^{m}$. Then, a reciprocally convex combination of these functions over $\mathbf{D}$ is a function of form

$$
\frac{1}{\alpha_{1}} \phi_{1}+\frac{1}{\alpha_{2}} \phi_{2}+\cdots+\frac{1}{\alpha_{N}} \phi_{N}: \mathbf{D} \longmapsto \mathbf{R}^{n},
$$

where the real numbers $\alpha_{i}$ satisfy $\alpha_{i}>0$ and $\sum_{i} \alpha_{i}=1$. 
Lemma 2.4 (see [1]). For any symmetric positive define matrix $R>0$, scalars $\gamma_{2}>\gamma_{1}>0$ and vector function $x:\left[\gamma_{1}, \gamma_{2}\right] \mapsto \mathbf{R}^{n}$ such that the integrations concerned are well defined, the following inequality holds

$$
-\left(\gamma_{2}-\gamma_{1}\right) \int_{\gamma_{1}}^{\gamma_{2}} x^{T}(s) R x(s) d s \leq-\int_{\gamma_{1}}^{\gamma_{2}} x^{T}(s) d s R \int_{\gamma_{1}}^{\gamma_{2}} x(s) d s
$$

Lemma 2.5 (see [11]). Let $f_{1}, f_{2}, \ldots, f_{N}: \mathbf{R}^{m} \mapsto \mathbf{R}$ have positive values in an open subset $\mathbf{D}$ of $\mathbf{R}^{m}$. Then, the reciprocally convex combination of $f_{i}$ over $\mathbf{D}$ satisfies

$$
\begin{aligned}
& \min _{\left\{\alpha_{i} \mid \alpha_{i}>0, \sum_{i} \alpha_{i}=1\right\}} \sum_{i} \frac{1}{\alpha_{i}} f_{i}(t)=\sum_{i} f_{i}(t)+\max _{g_{i, j}(t)} \sum_{i \neq j} g_{i, j}(t) \\
& \text { subject to }\left\{g_{i, j}: \mathbf{R}^{m} \mapsto \mathbf{R}, g_{j, i}(t) \triangleq g_{i, j}(t),\left[\begin{array}{cc}
f_{i}(t) & g_{i, j}(t) \\
g_{i, j}(t) & f_{j}(t)
\end{array}\right] \geq 0\right\} .
\end{aligned}
$$

\section{Main Results}

In this section, we consider the stability of singular time-delay system (2.1). For simplicity, we

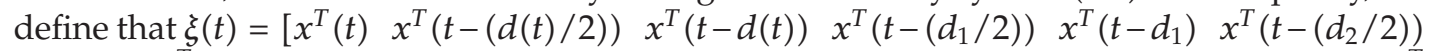
$\left.x^{T}\left(t-d_{2}\right)\right]^{T}, e_{i}(i=1,2, \ldots, 7)$ are block entry matrices, for example, $e_{3}=\left[\begin{array}{lllllll}0 & 0 & I & 0 & 0 & 0 & 0\end{array}\right]^{T}$ and $e_{8}=\left(A e_{1}^{T}+B e_{3}^{T}\right)^{T}$. Now, we provide a novel delay-dependent stability criterion for singular time-delay system (2.1) as follows.

Theorem 3.1. Given scalars $0<d_{1}<d_{2}$ and $0 \leq \mu<1$, for any delay $d(t)$ satisfying (2.2), singular time-delay system (2.1) is regular, impulse free, and stable if there exist matrices $P>0, Q_{i}=$ $\left[\begin{array}{l}Q_{i 1} \\ Q_{i 2} \\ Q_{i 2}^{T} Q_{i 3}\end{array}\right] \geq 0(i=1,2), Q_{3} \geq 0, Q_{4} \geq 0, R_{1} \geq 0, R_{2} \geq 0, R_{3} \geq 0, Z_{1}, Z_{2}$ and $S$, such that the following LMIs (3.1)-(3.3) hold

$$
\begin{aligned}
\Upsilon= & e_{1} E^{T} P e_{8}^{T}+e_{8} P E e_{1}^{T}+\left[\begin{array}{ll}
e_{1} & e_{4}
\end{array}\right] Q_{1}\left[\begin{array}{ll}
e_{1} & e_{4}
\end{array}\right]^{T}-\left[\begin{array}{ll}
e_{4} & e_{5}
\end{array}\right] Q_{1}\left[\begin{array}{ll}
e_{4} & e_{5}
\end{array}\right]^{T}+\left[\begin{array}{ll}
e_{1} & e_{6}
\end{array}\right] Q_{2}\left[\begin{array}{ll}
e_{1} & e_{6}
\end{array}\right]^{T} \\
& -\left[\begin{array}{ll}
e_{6} & e_{7}
\end{array}\right] Q_{2}\left[\begin{array}{ll}
e_{6} & e_{7}
\end{array}\right]^{T}+e_{1} Q_{3} e_{1}^{T}-\left(1-\frac{\mu}{2}\right) e_{2} Q_{3} e_{2}^{T}+e_{5} Q_{4} e_{5}^{T}-(1-\mu) e_{3} Q_{4} e_{3}^{T}+e_{8} \tilde{R} e_{8}^{T} \\
& -\left(e_{1}-e_{4}\right) E^{T} R_{1} E\left(e_{1}-e_{4}\right)^{T}+e_{1} S \Psi^{T} e_{8}^{T}+e_{8} \Psi S^{T} e_{1}^{T} \\
& -\left[\begin{array}{l}
e_{2}^{T}-e_{6}^{T} \\
e_{4}^{T}-e_{2}^{T}
\end{array}\right]^{T}\left[\begin{array}{ll}
E^{T} R_{2} E & E^{T} Z_{1} E \\
E^{T} Z_{1}^{T} E & E^{T} R_{2} E
\end{array}\right]\left[\begin{array}{l}
e_{2}^{T}-e_{6}^{T} \\
e_{4}^{T}-e_{2}^{T}
\end{array}\right] \\
& -\left[\begin{array}{l}
e_{3}^{T}-e_{7}^{T} \\
e_{5}^{T}-e_{3}^{T}
\end{array}\right]^{T}\left[\begin{array}{ll}
E^{T} R_{3} E & E^{T} Z_{2} E \\
E^{T} Z_{2}^{T} E & E^{T} R_{3} E
\end{array}\right]\left[\begin{array}{c}
e_{3}^{T}-e_{7}^{T} \\
e_{5}^{T}-e_{3}^{T}
\end{array}\right]<0
\end{aligned}
$$


Mathematical Problems in Engineering

$$
\begin{aligned}
& {\left[\begin{array}{ll}
R_{2} & Z_{1} \\
Z_{1}^{T} & R_{2}
\end{array}\right] \geq 0} \\
& {\left[\begin{array}{ll}
R_{3} & Z_{2} \\
Z_{2}^{T} & R_{3}
\end{array}\right] \geq 0,}
\end{aligned}
$$

where $\widetilde{R}=\left(d_{1} / 2\right)^{2} R_{1}+\left(\left(d_{2}-d_{1}\right) / 2\right)^{2} R_{2}+\left(d_{2}-d_{1}\right)^{2} R_{3}$, and $\Psi \in \mathbf{R}^{n \times(n-r)}$ is any full-column rank matrix satisfying $E^{T} \Psi=0$.

Proof. The proof is divided into two parts. The first part deals with the regularity and impulsefree properties, and the second part treats the stability property of the studied class of systems. First of all, we show that the singular time-delay system (2.1) is regular and impulse free for any time-delay $d(t)$ satisfying (2.2). From LMI (3.1), it follows that

$$
\left[\begin{array}{cccc}
\Xi_{11} & E^{T} P B+S \Psi^{T} B & Q_{12}+E^{T} R_{1} E & 0 \\
B^{T} P E+B^{T} \Psi S^{T} & \Xi_{22} & 0 & E^{T} R_{3} E-E^{T} Z_{2} E \\
Q_{12}^{T}+E^{T} R_{1} E & 0 & \Xi_{33} & -Q_{12} \\
0 & E^{T} R_{3} E-E^{T} Z_{2}^{T} E & -Q_{12}^{T} & -Q_{13}+Q_{4}-E^{T} R_{3} E
\end{array}\right]<0
$$

where

$$
\begin{gathered}
\Xi_{11}=E^{T} P A+A^{T} P E+Q_{11}+Q_{21}+Q_{3}-E^{T} R_{1} E+S \Psi^{T} A+A^{T} \Psi S^{T}, \\
\Xi_{22}=-(1-\mu) Q_{4}-2 E^{T} R_{3} E+E^{T} Z_{2} E+E^{T} Z_{2}^{T} E, \\
\Xi_{33}=Q_{13}-Q_{11}-E^{T} R_{1} E-E^{T} R_{2} E .
\end{gathered}
$$

From LMI (3.4), it easy to see that $\Xi_{11}<0$, using the fact that $Q_{11} \geq 0, Q_{21} \geq 0$ and $Q_{3} \geq 0$, we have

$$
E^{T} P A+A^{T} P E-E^{T} R_{1} E+S \Psi^{T} A+A^{T} \Psi S^{T}<0 .
$$

Since rank $E=r \leq n$, there must exist two invertible matrices $G, H \in \mathbf{R}^{n \times n}$ such that

$$
G E H=\left[\begin{array}{cc}
I_{r} & 0 \\
0 & 0
\end{array}\right]
$$

Set

$$
G A H=\left[\begin{array}{ll}
A_{11} & A_{12} \\
A_{21} & A_{22}
\end{array}\right], \quad G^{-T} \Psi=\left[\begin{array}{c}
0 \\
\tilde{\Psi}
\end{array}\right], \quad H^{T} S=\left[\begin{array}{l}
S_{1} \\
S_{2}
\end{array}\right],
$$

where $\widetilde{\Psi} \in \mathbf{R}^{(n-r) \times(n-r)}$ is a nonsingular matrix. Pre-multiplying and post-multiplying (3.6) by $H^{T}$ and $H$, respectively, we can easily formulate the following inequality:

$$
\left[\begin{array}{cc}
\Theta_{11} & \Theta_{12} \\
\Theta_{12}^{T} & A_{22}^{T} \tilde{\Psi} S_{2}^{T}+S_{2} \tilde{\Psi}^{T} A_{22}
\end{array}\right]<0,
$$


where $\Theta_{11}$ and $\Theta_{12}$ are not relevant in the following discussion; the real expression of these two matrices are omitted here. From (3.9), it is easy to see that

$$
A_{22}^{T} \tilde{\Psi} S_{2}^{T}+S_{2} \tilde{\Psi}^{T} A_{22}<0,
$$

which implies that matrix $A_{22}$ is nonsingular. Otherwise, supposing that $A_{22}$ is singular, there must exist a nonzero vector $\eta \in \mathbf{R}^{n-r}$, which ensures that $A_{22} \eta=0$. And then we can conclude that $\eta^{T}\left(A_{22}^{T} \tilde{\Psi} S_{2}^{T}+S_{2} \tilde{\Psi}^{T} A_{22}\right) \eta=0$, and this contradicts (3.10). So $A_{22}$ is nonsingular, which implies that the pair $(E, A)$ is regular and impulse free [16].

On the other hand, Pre-multiplying and post-multiplying (3.4) by $\left[\begin{array}{llll}I & I & I & I\end{array}\right]$ and $\left[\begin{array}{llll}I & I & I & I\end{array}\right]^{T}$, respectively, yields

$$
\begin{aligned}
& E^{T} P(A+B)+(A+B)^{T} P E-E^{T}\left(R_{2}+R_{3}\right) E+S \Psi^{T}(A+B)+(A+B)^{T} \Psi S^{T} \\
& \quad+Q_{21}+Q_{3}+\mu Q_{4}<0 .
\end{aligned}
$$

From (3.11), taking conditions $Q_{21} \geq 0, Q_{3} \geq 0, Q_{4} \geq 0$ and $\mu \geq 0$ into account, we obtain

$$
E^{T} P(A+B)+(A+B)^{T} P E-E^{T}\left(R_{2}+R_{3}\right) E+S \Psi^{T}(A+B)+(A+B)^{T} \Psi S^{T}<0
$$

Proceeding in a similar manner as above, we can find (3.12) implies that the pair $(E, A+B)$ is regular and impulse free. Thus, according to Definition 2.2, singular time-delay system (2.1) is regular and impulse free for any time-delay $d(t)$ satisfying (2.2).

In the following, we will prove that singular delay-delay system (2.1) is stable. Construct a new class Lyapunov functional for system (2.1) as follows:

$$
V(t)=V_{1}(t)+V_{2}(t)+V_{3}(t)
$$

where

$$
\begin{gathered}
V_{1}(t)=x^{T}(t) E^{T} P E x(t), \\
V_{2}(t)=\int_{t-d_{1} / 2}^{t} \xi_{1}^{T}(s) Q_{1} \xi_{1}(s) d s+\int_{t-d_{2} / 2}^{t} \xi_{2}^{T}(s) Q_{2} \xi_{2}(s) d s \\
+\int_{t-d(t) / 2}^{t} x^{T}(s) Q_{3} x(s) d s+\int_{t-d(t)}^{t-d_{1}} x^{T}(s) Q_{4} x(s) d s, \\
V_{3}(t)=\frac{d_{1}}{2} \int_{-d_{1} / 2}^{0} \int_{t+\alpha}^{t} \dot{x}^{T}(\beta) E^{T} R_{1} E \dot{x}(\beta) d \beta d \alpha+\frac{d_{2}-d_{1}}{2} \int_{-d_{2} / 2}^{-d_{1} / 2} \int_{t+\alpha}^{t} \dot{x}^{T}(\beta) E^{T} R_{2} E \dot{x}(\beta) d \beta d \alpha \\
+\left(d_{2}-d_{1}\right) \int_{-d_{2}}^{-d_{1}} \int_{t+\alpha}^{t} \dot{x}^{T}(\beta) E^{T} R_{3} E \dot{x}(\beta) d \beta d \alpha,
\end{gathered}
$$


with

$$
\xi_{1}(s)=\left[\begin{array}{ll}
x^{T}(s) & x^{T}\left(s-\frac{d_{1}}{2}\right)
\end{array}\right]^{T}, \quad \xi_{2}(s)=\left[\begin{array}{ll}
x^{T}(s) & x^{T}\left(s-\frac{d_{2}}{2}\right)
\end{array}\right]^{T}
$$

It is easy to see that

$$
\dot{V}(t)=\dot{V}_{1}(t)+\dot{V}_{2}(t)+\dot{V}_{3}(t)
$$

The time derivative of each $V_{i}(t)(i=1,2,3)$ along trajectories of the singular time-delay system (2.1) can be processed as

$$
\begin{aligned}
& \dot{V}_{1}(t)=2 x^{T}(t) E^{T} P E \dot{x}(t)=2 \xi^{T}(t) e_{1} E^{T} P e_{8}^{T} \xi(t), \\
& \dot{V}_{2}(t)= \xi_{1}^{T}(t) Q_{1} \xi_{1}(t)-\xi_{1}^{T}\left(t-\frac{d_{1}}{2}\right) Q_{1} \xi_{1}\left(t-\frac{d_{1}}{2}\right)+\xi_{2}^{T}(t) Q_{2} \xi_{2}(t)-\xi_{2}^{T}\left(t-\frac{d_{2}}{2}\right) Q_{2} \xi_{2}\left(t-\frac{d_{2}}{2}\right) \\
&+ x^{T}(t) Q_{3} x(t)-\left(1-\frac{\dot{d}(t)}{2}\right) x^{T}\left(t-\frac{d(t)}{2}\right) Q_{3} x\left(t-\frac{d(t)}{2}\right) \\
&+x^{T}\left(t-d_{1}\right) Q_{4} x\left(t-d_{1}\right)-(1-\dot{d}(t)) x^{T}(t-d(t)) Q_{4} x(t-d(t)) \\
& \leqslant \xi^{T}(t)\left(\left[e_{1} e_{4}\right] Q_{1}\left[e_{1} e_{4}\right]^{T}-\left[e_{4} e_{5}\right] Q_{1}\left[e_{4} e_{5}\right]^{T}+\left[e_{1} e_{6}\right] Q_{2}\left[e_{1} e_{6}\right]^{T}\right. \\
&\left.-\left[e_{6} e_{7}\right] Q_{2}\left[e_{6} e_{7}\right]^{T}+e_{1} Q_{3} e_{1}^{T}-\left(1-\frac{\mu}{2}\right) e_{2} Q_{3} e_{2}^{T}+e_{5} Q_{4} e_{5}^{T}-(1-\mu) e_{3} Q_{4} e_{3}^{T}\right) \xi(t), \\
& \dot{V}_{3}(t)= \dot{x}^{T}(t) E^{T} \tilde{R} E \dot{x}(t)-\frac{d_{1}}{2} \int_{t-d_{1} / 2}^{t} \dot{x}^{T}(s) E^{T} R_{1} E \dot{x}(s) d s \\
&-\frac{d_{2}-d_{1}}{2} \int_{t-d_{2} / 2}^{t-d_{1} / 2} \dot{x}^{T}(s) E^{T} R_{2} E \dot{x}(s) d s-\left(d_{2}-d_{1}\right) \int_{t-d_{2}}^{t-d_{1}} \dot{x}^{T}(s) E^{T} R_{3} E \dot{x}(s) d s \\
&= \dot{x}^{T}(t) E^{T} \tilde{R} E \dot{x}(t)-\frac{d_{1}}{2} \int_{t-d_{1} / 2}^{t} \dot{x}^{T}(s) E^{T} R_{1} E \dot{x}(s) d s \\
&-\frac{d_{2}-d_{1}}{2} \int_{t-d_{2} / 2}^{t-d(t) / 2} \dot{x}^{T}(s) E^{T} R_{2} E \dot{x}(s) d s-\frac{d_{2}-d_{1}}{2} \int_{t-d(t) / 2}^{t-d_{1} / 2} \dot{x}^{T}(s) E^{T} R_{2} E \dot{x}(s) d s \\
&-\left(d_{2}-d_{1}\right) \int_{t-d_{2}}^{t-d(t)} \dot{x}^{T}(s) E^{T} R_{3} E \dot{x}(s) d s-\left(d_{2}-d_{1}\right) \int_{t-d(t)}^{t-d_{1}} \dot{x}^{T}(s) E^{T} R_{3} E \dot{x}(s) d s .
\end{aligned}
$$


Applying Lemma 2.4 to the last five integral terms, we can obtain

$$
\begin{aligned}
\dot{V}_{3}(t) \leqslant & \xi^{T}(t) e_{8} \widetilde{R} e_{8}^{T} \xi(t)-\xi^{T}(t)\left(e_{1}-e_{4}\right) E^{T} R_{1} E\left(e_{1}-e_{4}\right)^{T} \xi(t)-\frac{1}{\alpha_{1}} f_{1}(t)-\frac{1}{\alpha_{2}} f_{2}(t) \\
& -\frac{1}{\alpha_{1}} g_{1}(t)-\frac{1}{\alpha_{2}} g_{2}(t)
\end{aligned}
$$

where

$$
\begin{aligned}
\alpha_{1}=\frac{d_{2}-d(t)}{d_{2}-d_{1}}, & \alpha_{2}=\frac{d(t)-d_{1}}{d_{2}-d_{1}}, \\
f_{1}(t)=\xi^{T}(t)\left(e_{2}-e_{6}\right) E^{T} R_{2} E\left(e_{2}-e_{6}\right)^{T} \xi(t), & f_{2}(t)=\xi^{T}(t)\left(e_{4}-e_{2}\right) E^{T} R_{2} E\left(e_{4}-e_{2}\right)^{T} \xi(t), \\
g_{1}(t)=\xi^{T}(t)\left(e_{3}-e_{7}\right) E^{T} R_{3} E\left(e_{3}-e_{7}\right)^{T} \xi(t), & g_{2}(t)=\xi^{T}(t)\left(e_{5}-e_{3}\right) E^{T} R_{3} E\left(e_{5}-e_{3}\right)^{T} \xi(t) .
\end{aligned}
$$

Pre-multiplying and post-multiplying LMI (3.2) by $\operatorname{diag}\left\{\xi^{T}(t)\left(e_{2}-e_{6}\right) E^{T}, \xi^{T}(t)\left(e_{4}-e_{2}\right) E^{T}\right\}$ and $\operatorname{diag}\left\{E\left(e_{2}-e_{6}\right)^{T} \xi(t), E\left(e_{4}-e_{2}\right)^{T} \xi(t)\right\}$, respectively, we have

$$
\left[\begin{array}{ll}
f_{1}(t) & f_{1,2}(t) \\
f_{2,1}(t) & f_{2}(t)
\end{array}\right] \geq 0
$$

where

$$
\begin{aligned}
& f_{1,2}(t)=\xi^{T}(t)\left(e_{2}-e_{6}\right) E^{T} Z_{1} E\left(e_{4}-e_{2}\right)^{T} \xi(t) \\
& f_{2,1}(t)=\xi^{T}(t)\left(e_{4}-e_{2}\right) E^{T} Z_{1}^{T} E\left(e_{2}-e_{6}\right)^{T} \xi(t) .
\end{aligned}
$$

By using Lemma 2.5, we have

$$
\begin{aligned}
-\frac{1}{\alpha_{1}} f_{1}(t)-\frac{1}{\alpha_{2}} f_{2}(t) & \leq-f_{1}(t)-f_{2}(t)-f_{1,2}(t)-f_{2,1}(t) \\
& =-\xi^{T}(t)\left[\begin{array}{l}
e_{2}^{T}-e_{6}^{T} \\
e_{4}^{T}-e_{2}^{T}
\end{array}\right]^{T}\left[\begin{array}{ll}
E^{T} R_{2} E & E^{T} Z_{1} E \\
E^{T} Z_{1}^{T} E & E^{T} R_{2} E
\end{array}\right]\left[\begin{array}{l}
e_{2}^{T}-e_{6}^{T} \\
e_{4}^{T}-e_{2}^{T}
\end{array}\right] \xi(t) .
\end{aligned}
$$

Similarly, from LMI (3.3), we can get

$$
-\frac{1}{\alpha_{1}} g_{1}(t)-\frac{1}{\alpha_{2}} g_{2}(t) \leq-\xi^{T}(t)\left[\begin{array}{c}
e_{3}^{T}-e_{7}^{T} \\
e_{5}^{T}-e_{3}^{T}
\end{array}\right]^{T}\left[\begin{array}{cc}
E^{T} R_{3} E & E^{T} Z_{2} E \\
E^{T} Z_{2}^{T} E & E^{T} R_{3} E
\end{array}\right]\left[\begin{array}{c}
e_{3}^{T}-e_{7}^{T} \\
e_{5}^{T}-e_{3}^{T}
\end{array}\right] \xi(t) .
$$


Combining (3.20)-(3.25), we can furthermore get

$$
\begin{aligned}
\dot{V}_{3}(t) \leq & \xi^{T}(t) e_{8} \tilde{R} e_{8}^{T} \xi(t)-\xi^{T}(t)\left(e_{1}-e_{4}\right) E^{T} R_{1} E\left(e_{1}-e_{4}\right)^{T} \xi(t) \\
& -\xi^{T}(t)\left[\begin{array}{l}
e_{2}^{T}-e_{6}^{T} \\
e_{4}^{T}-e_{2}^{T}
\end{array}\right]^{T}\left[\begin{array}{ll}
E^{T} R_{2} E & E^{T} Z_{1} E \\
E^{T} Z_{1}^{T} E & E^{T} R_{2} E
\end{array}\right]\left[\begin{array}{l}
e_{2}^{T}-e_{6}^{T} \\
e_{4}^{T}-e_{2}^{T}
\end{array}\right] \xi(t) \\
& -\xi^{T}(t)\left[\begin{array}{l}
e_{3}^{T}-e_{7}^{T} \\
e_{5}^{T}-e_{3}^{T}
\end{array}\right]^{T}\left[\begin{array}{ll}
E^{T} R_{3} E & E^{T} Z_{2} E \\
E^{T} Z_{2}^{T} E & E^{T} R_{3} E
\end{array}\right]\left[\begin{array}{l}
e_{3}^{T}-e_{7}^{T} \\
e_{5}^{T}-e_{3}^{T}
\end{array}\right] \xi(t) .
\end{aligned}
$$

Note that when $d(t)=d_{1}$ or $d(t)=d_{2}$, we have $\xi^{T}(t)\left(e_{4}-e_{2}\right)=\xi^{T}(t)\left(e_{5}-e_{3}\right)=0$ or $\xi^{T}(t)\left(e_{2}-\right.$ $\left.e_{6}\right)=\xi^{T}(t)\left(e_{3}-e_{7}\right)=0$, respectively. So relation (3.26) still holds.

Noting $E^{T} \Psi=0$, we have

$$
0=2 x^{T}(t) S \Psi^{T} E \dot{x}(t)=2 \xi^{T}(t) e_{1} S \Psi^{T} e_{8}^{T} \xi(t),
$$

where $S$ is any matrix with appropriate dimensions.

Adding (3.27) to the right of (3.16) and substituting (3.17), (3.18), and (3.26) into (3.16), we have

$$
\dot{V}(t) \leq \xi^{T}(t) \Upsilon \xi(t) .
$$

From LMI (3.1), it is easy to see that $\dot{V}(t)<0$ for any $\xi(t) \neq 0$. Hence, there exists a sufficiently small positive scalar $\varepsilon>0$, such that

$$
\dot{V}(t) \leq-\varepsilon\|x(t)\|^{2}
$$

By (3.29), the following steps are similar to the proof of Proposition 1 in [35] and Theorem 1 in [36], we can deduce that singular time-delay system (2.1) is stable. This completes our proof.

Remark 3.2. Based on the new Lyapunov functional in (3.13), together with the reciprocally convex approach and LMI technique, Theorem 3.1 proposed a delay-dependent criterion guaranteeing the considered singular time-delay system to be regular, impulse free, and stable. Lyapunov functional (3.13) is constructed by using the idea of "delay-partitioning" $[25,37,39]$. We consider the lower bound $d_{1}$, upper bound $d_{2}$, and time-varying delay $d(t)$ in our Lyapunov functional by dividing them into two equal segments, such that the information of delayed states $d_{1} / 2, d_{2} / 2$ and $d(t) / 2$ are all taken into account. Therefore, the criterion in Theorem 3.1 is expected to be less conservative than some previous ones, which will be demonstrated in the sequel. 
If the matrix $E$ is nonsingular, then the stability problem of singular system (2.1) is reduced to analyzing the stability of the regular system:

$$
\begin{aligned}
& \dot{x}(t)=A x(t)+B x(t-d(t)), \\
& x(\theta)=\varphi(\theta), \quad \theta \in\left[-d_{2}, 0\right] .
\end{aligned}
$$

This problem has been widely studied in the recent literature (see, e.g., [3-14]). We choose Lyapunov functional:

$$
\tilde{V}(t)=\tilde{V}_{1}(t)+V_{2}(t)+\tilde{V}_{3}(t),
$$

where

$$
\begin{gathered}
\tilde{V}_{1}(t)=x^{T}(t) P x(t), \\
\tilde{V}_{3}(t)=\frac{d_{1}}{2} \int_{-d_{1} / 2}^{0} \int_{t+\alpha}^{t} \dot{x}^{T}(\beta) R_{1} \dot{x}(\beta) d \beta d \alpha+\frac{d_{2}-d_{1}}{2} \int_{-d_{2} / 2}^{-d_{1} / 2} \int_{t+\alpha}^{t} \dot{x}^{T}(\beta) R_{2} \dot{x}(\beta) d \beta d \alpha \\
+\left(d_{2}-d_{1}\right) \int_{-d_{2}}^{-d_{1}} \int_{t+\alpha}^{t} \dot{x}^{T}(\beta) R_{3} \dot{x}(\beta) d \beta d \alpha,
\end{gathered}
$$

and $V_{2}(t)$ is defined in (3.13).

By employing the Lyapunov functional (3.31) and using the similar proof of Theorem 3.1, we can obtain the following delay-dependent stability criterion for time-delay system (3.30).

Corollary 3.3. Given scalars $0<d_{1}<d_{2}$ and $0 \leq \mu<1$, for any delay $d(t)$ satisfying (2.2), timedelay system (3.30) is stable if there exist matrices $P>0, Q_{i}=\left[\begin{array}{cc}Q_{i 1} & Q_{i 2} \\ Q_{i 2}^{T} & Q_{i 3}\end{array}\right] \geq 0(i=1,2), Q_{3} \geq 0$, $Q_{4} \geq 0, R_{1} \geq 0, R_{2} \geq 0, R_{3} \geq 0$ and $Z_{1}, Z_{2}$, such that LMIs (3.2), (3.3), and following LMI (3.34) hold

$$
\begin{aligned}
& e_{1} P e_{8}^{T}+e_{8} P e_{1}^{T}+\left[\begin{array}{ll}
e_{1} & e_{4}
\end{array}\right] Q_{1}\left[\begin{array}{ll}
e_{1} & e_{4}
\end{array}\right]^{T}-\left[\begin{array}{ll}
e_{4} & e_{5}
\end{array}\right] Q_{1}\left[\begin{array}{ll}
e_{4} & e_{5}
\end{array}\right]^{T} \\
&+ {\left[\begin{array}{ll}
e_{1} & e_{6}
\end{array}\right] Q_{2}\left[\begin{array}{ll}
e_{1} & e_{6}
\end{array}\right]^{T}-\left[\begin{array}{ll}
e_{6} & e_{7}
\end{array}\right] Q_{2}\left[\begin{array}{ll}
e_{6} & e_{7}
\end{array}\right]^{T} } \\
&+e_{1} Q_{3} e_{1}^{T}-\left(1-\frac{\mu}{2}\right) e_{2} Q_{3} e_{2}^{T}+e_{5} Q_{4} e_{5}^{T}-(1-\mu) e_{3} Q_{4} e_{3}^{T}+e_{8} \tilde{R} e_{8}^{T}-\left(e_{1}-e_{4}\right) R_{1}\left(e_{1}-e_{4}\right)^{T} \\
&-\left[\begin{array}{l}
e_{2}^{T}-e_{6}^{T} \\
e_{4}^{T}-e_{2}^{T}
\end{array}\right]^{T}\left[\begin{array}{ll}
R_{2} & Z_{1} \\
Z_{1}^{T} & R_{2}
\end{array}\right]\left[\begin{array}{l}
e_{2}^{T}-e_{6}^{T} \\
e_{4}^{T}-e_{2}^{T}
\end{array}\right]-\left[\begin{array}{c}
e_{3}^{T}-e_{7}^{T} \\
e_{5}^{T}-e_{3}^{T}
\end{array}\right]^{T}\left[\begin{array}{ll}
R_{3} & Z_{2} \\
Z_{2}^{T} & R_{3}
\end{array}\right]\left[\begin{array}{l}
e_{3}^{T}-e_{7}^{T} \\
e_{5}^{T}-e_{3}^{T}
\end{array}\right]<0,
\end{aligned}
$$

where matrix $\widetilde{R}$ is defined in (3.1). 
Remark 3.4. As mentioned in the introduction, through the use of free-weighting matrices [5] or the introduction of the Lyapunov functional with triple-integral terms [13, 14], we can derive less conservative stability criteria for system (3.30), but it makes the criteria mathematically complex and computationally less effective. In this paper, the Lyapunov functional (3.31) does not contain any triple-integral terms, and when estimating $\dot{V}(t)$, we have not introduced free-weighting matrix. From a mathematical point of view, it is simple. Meanwhile, Corollary 3.3 in this paper is less conservative than the results in $[5,13,14]$, which will be demonstrated in the sequel.

Theorem 3.1 and Corollary 3.3 give new stability criteria of system (2.1) and system (3.30) with $d(t)$ satisfying (2.2), respectively. They can be applied to both slow and fast timevarying delays only if $0 \leq \mu<1$ is known. In many circumstances, the information of the time derivative of delay $\mu$ is unknown or the time derivative of delay is known but $\mu \geq 1$. Regarding this case, the delay-dependent and rate-independent criteria can be derived by choosing $Q_{3}=Q_{4}=0$ in Theorem 3.1 and Corollary 3.3, respectively. Therefore, we have the following Corollaries 3.5 and 3.6.

Corollary 3.5. Given scalars $0<d_{1}<d_{2}$, for any delay $d(t)$ satisfying $d_{1} \leq d(t) \leq d_{2}$, singular system (2.1) is regular, impulse free, and stable if there exist matrices $P>0, Q_{i}=\left[\begin{array}{ll}Q_{i 1} & Q_{i 2} \\ Q_{i 2}^{T} & Q_{i 3}\end{array}\right] \geq 0, Z_{i}(i=$ $1,2), R_{j} \geq 0(j=1,2,3)$ and $S$, such that LMIs (3.2), (3.3), and following LMI (3.35) hold

$$
\begin{aligned}
e_{1} & E^{T} P e_{8}^{T}+e_{8} P E e_{1}^{T}+\left[\begin{array}{ll}
e_{1} & e_{4}
\end{array}\right] Q_{1}\left[\begin{array}{ll}
e_{1} & e_{4}
\end{array}\right]^{T}-\left[\begin{array}{ll}
e_{4} & e_{5}
\end{array}\right] Q_{1}\left[\begin{array}{ll}
e_{4} & e_{5}
\end{array}\right]^{T}+\left[\begin{array}{ll}
e_{1} & e_{6}
\end{array}\right] Q_{2}\left[\begin{array}{ll}
e_{1} & e_{6}
\end{array}\right]^{T} \\
- & {\left[\begin{array}{ll}
e_{6} & e_{7}
\end{array}\right] Q_{2}\left[\begin{array}{ll}
e_{6} & e_{7}
\end{array}\right]^{T}+e_{8} \widetilde{R} e_{8}^{T}-\left(e_{1}-e_{4}\right) E^{T} R_{1} E\left(e_{1}-e_{4}\right)^{T}+e_{1} S \Psi^{T} e_{8}^{T}+e_{8} \Psi S^{T} e_{1}^{T} } \\
& -\left[\begin{array}{l}
e_{2}^{T}-e_{6}^{T} \\
e_{4}^{T}-e_{2}^{T}
\end{array}\right]^{T}\left[\begin{array}{ll}
E^{T} R_{2} E & E^{T} Z_{1} E \\
E^{T} Z_{1}^{T} E & E^{T} R_{2} E
\end{array}\right]\left[\begin{array}{l}
e_{2}^{T}-e_{6}^{T} \\
e_{4}^{T}-e_{2}^{T}
\end{array}\right] \\
& -\left[\begin{array}{c}
e_{3}^{T}-e_{7}^{T} \\
e_{5}^{T}-e_{3}^{T}
\end{array}\right]^{T}\left[\begin{array}{ll}
E^{T} R_{3} E & E^{T} Z_{2} E \\
E^{T} Z_{2}^{T} E & E^{T} R_{3} E
\end{array}\right]\left[\begin{array}{c}
e_{3}^{T}-e_{7}^{T} \\
e_{5}^{T}-e_{3}^{T}
\end{array}\right]<0,
\end{aligned}
$$

where matrices $\widetilde{R}$ and $\Psi$ are defined in (3.1).

Corollary 3.6. Given scalars $0<d_{1}<d_{2}$, for any delay $d(t)$ satisfying $d_{1} \leq d(t) \leq d_{2}$, time-delay system (3.30) is stable if there exist matrices $P>0, Q_{i}=\left[\begin{array}{cc}Q_{i 1} & Q_{i 2} \\ Q_{i 2}^{T} & Q_{i 3}\end{array}\right] \geq 0, Z_{i}(i=1,2)$ and $R_{j} \geq 0(j=$ 1,2,3), such that LMIs (3.2), (3.3), and following LMI (3.36) hold

$$
\begin{aligned}
e_{1} P e_{8}^{T}+e_{8} P e_{1}^{T}+\left[\begin{array}{ll}
e_{1} & e_{4}
\end{array}\right] Q_{1}\left[\begin{array}{ll}
e_{1} & e_{4}
\end{array}\right]^{T}-\left[\begin{array}{ll}
e_{4} & e_{5}
\end{array}\right] Q_{1}\left[\begin{array}{ll}
e_{4} & e_{5}
\end{array}\right]^{T}+\left[\begin{array}{ll}
e_{1} & e_{6}
\end{array}\right] Q_{2}\left[\begin{array}{ll}
e_{1} & e_{6}
\end{array}\right]^{T} \\
-\left[\begin{array}{ll}
e_{6} & e_{7}
\end{array}\right] Q_{2}\left[\begin{array}{ll}
e_{6} & e_{7}
\end{array}\right]^{T}+e_{8} \widetilde{R} e_{8}^{T}-\left(e_{1}-e_{4}\right) R_{1}\left(e_{1}-e_{4}\right)^{T} \\
-\left[\begin{array}{l}
e_{2}^{T}-e_{6}^{T} \\
e_{4}^{T}-e_{2}^{T}
\end{array}\right]^{T}\left[\begin{array}{ll}
R_{2} & Z_{1} \\
Z_{1}^{T} & R_{2}
\end{array}\right]\left[\begin{array}{l}
e_{2}^{T}-e_{6}^{T} \\
e_{4}^{T}-e_{2}^{T}
\end{array}\right]-\left[\begin{array}{l}
e_{3}^{T}-e_{7}^{T} \\
e_{5}^{T}-e_{3}^{T}
\end{array}\right]^{T}\left[\begin{array}{ll}
R_{3} & Z_{2} \\
Z_{2}^{T} & R_{3}
\end{array}\right]\left[\begin{array}{l}
e_{3}^{T}-e_{7}^{T} \\
e_{5}^{T}-e_{3}^{T}
\end{array}\right]<0,
\end{aligned}
$$

where matrix $\widetilde{R}$ is defined in (3.1). 
Remark 3.7. By employing similar method and choosing $Q_{1}=0, R_{1}=0$ in Lyapunov functionals (3.13) and (3.31), all the above results obtained in this paper can easily extend to delay-dependent stability results for systems (2.1) and (3.30) with $d_{1}=0$. As for example, we can obtain a delay-dependent stability criterion using similar method of Theorem 3.1 for system (2.1) with $d_{1}=0$, and $d(t)=d$ is a constant time delay. The obtained stability criterion for this case is shown in the following Corollary 3.8.

Corollary 3.8. Given scalar $d_{2}>0$, for any constant time delay $d(t)=d$ satisfying $0 \leq d \leq d_{2}$, singular time-delay system (2.1) is regular, impulse free, and stable if there exist matrices $P>0, Q_{2}=$ $\left[\begin{array}{ll}Q_{21} & Q_{22} \\ Q_{22}^{T} & Q_{23}\end{array}\right] \geq 0, Q_{3} \geq 0, Q_{4} \geq 0, R_{2} \geq 0, R_{3} \geq 0, Z_{1}, Z_{2}$ and $S$, such that LMIs (3.2),(3.3), and following LMI (3.37) hold

$$
\begin{aligned}
\bar{e}_{1} & E^{T} P \bar{e}_{6}^{T}+\bar{e}_{6} P E \bar{e}_{1}^{T}+\left[\begin{array}{ll}
\bar{e}_{1} & \bar{e}_{4}
\end{array}\right] Q_{2}\left[\begin{array}{ll}
\bar{e}_{1} & \bar{e}_{4}
\end{array}\right]^{T}-\left[\begin{array}{ll}
\bar{e}_{4} & \bar{e}_{5}
\end{array}\right] Q_{2}\left[\begin{array}{ll}
\bar{e}_{4} & \bar{e}_{5}
\end{array}\right]^{T}+\bar{e}_{1}\left(Q_{3}+Q_{4}\right) \bar{e}_{1}^{T} \\
& -\bar{e}_{2} Q_{3} \bar{e}_{2}^{T}-\bar{e}_{4} Q_{4} \bar{e}_{4}^{T}+\bar{e}_{6} \bar{R}_{\bar{e}} \bar{e}_{6}^{T}+\bar{e}_{1} S \Psi^{T} \bar{e}_{6}^{T}+\bar{e}_{6} \Psi S^{T} \bar{e}_{1}^{T} \\
& -\left[\begin{array}{c}
\bar{e}_{2}^{T}-\bar{e}_{4}^{T} \\
\bar{e}_{1}^{T}-\bar{e}_{2}^{T}
\end{array}\right]^{T}\left[\begin{array}{ll}
E^{T} R_{2} E & E^{T} Z_{1} E \\
E^{T} Z_{1}^{T} E & E^{T} R_{2} E
\end{array}\right]\left[\begin{array}{l}
\bar{e}_{2}^{T}-\bar{e}_{4}^{T} \\
\bar{e}_{1}^{T}-\bar{e}_{2}^{T}
\end{array}\right] \\
& -\left[\begin{array}{c}
\bar{e}_{3}^{T}-\bar{e}_{5}^{T} \\
\bar{e}_{1}^{T}-\bar{e}_{3}^{T}
\end{array}\right]^{T}\left[\begin{array}{ll}
E^{T} R_{3} E & E^{T} Z_{2} E \\
E^{T} Z_{2}^{T} E & E^{T} R_{3} E
\end{array}\right]\left[\begin{array}{l}
\bar{e}_{3}^{T}-\bar{e}_{5}^{T} \\
\bar{e}_{1}^{T}-\bar{e}_{3}^{T}
\end{array}\right]<0
\end{aligned}
$$

where $\bar{R}=\left(d_{2} / 2\right)^{2} R_{2}+d_{2}^{2} R_{3}, \Psi \in \mathbf{R}^{n \times(n-r)}$ is any full-column rank matrix satisfying $E^{T} \Psi=0$, and $\bar{e}_{i}(i=1,2, \ldots, 5)$ are block entry matrices, for example, $\bar{e}_{2}=\left[\begin{array}{lllll}0 & I & 0 & 0 & 0\end{array}\right]^{T}$, and $\bar{e}_{6}=\left(A \bar{e}_{1}^{T}+B \bar{e}_{3}^{T}\right)^{T}$.

Remark 3.9. It is worth pointing out that the obtained results in this paper are formulated in terms of LMIs, they can be easily solved using any LMI toolbox like one of Matlab or the one of Scilab.

\section{Numerical Examples}

In this section, we use three examples and compare our results with the previous ones to show the effectiveness of ours.

Example 4.1. Consider the singular time-delay system (2.1) with

$$
E=\left[\begin{array}{ll}
1 & 0 \\
0 & 0
\end{array}\right], \quad A=\left[\begin{array}{cc}
0.5 & 0 \\
0 & -1
\end{array}\right], \quad B=\left[\begin{array}{cc}
-1.1 & 1 \\
0 & 0.5
\end{array}\right]
$$

For various $\mu$, the allowable upper bounds $d_{2}$, which guarantee regular, impulse free, and stable of system (2.1) for given lower bounds $d_{1}$, are listed in Table 1. From Table 1, it can be seen that the stability criterion in Theorem 3.1 is less conservative than that in [35]. Especially, when $d_{1}=1.1$, the result in [35] is not feasible while the allowable upper bounds $d_{2}$ can also be obtained from Theorem 3.1 in this paper. 
Table 1: Allowable upper bound $d_{2}$ with given $d_{1}$ for different $\mu$.

\begin{tabular}{lccccccc}
\hline$d_{1}$ & Methods & $d_{1}=0.1$ & $d_{1}=0.3$ & $d_{1}=0.5$ & $d_{1}=0.7$ & $d_{1}=0.9$ & $d_{1}=1.1$ \\
\hline$\mu=0.1$ & [35] & 1.0494 & 1.0497 & 1.0504 & 1.0518 & 1.0565 & - \\
& Theorem 3.1 & 1.0769 & 1.0860 & 1.0887 & 1.0872 & 1.0873 & 1.1066 \\
$\mu=0.3$ & {$[35]$} & 1.0277 & 1.0315 & 1.0379 & 1.0466 & 1.0565 & - \\
& Theorem 3.1 & 1.0598 & 1.0615 & 1.0645 & 1.0726 & 1.0865 & 1.1066 \\
$\mu=0.5$ & [35] & 1.0235 & 1.0303 & 1.0379 & 1.0466 & 1.0565 & - \\
& Theorem 3.1 & 1.0598 & 1.0607 & 1.0644 & 1.0726 & 1.0865 & 1.1066 \\
$\mu=0.7$ & [35] & 1.0235 & 1.0303 & 1.0379 & 1.0466 & 1.0565 & - \\
& Theorem 3.1 & 1.0598 & 1.0607 & 1.0644 & 1.0726 & 1.0865 & 1.1066 \\
\hline
\end{tabular}

Table 2: Allowable upper bound $d_{2}$ with given $d_{1}$ for different $\mu$.

\begin{tabular}{ccccccc}
\hline$\mu$ & Methods & $d_{1}=1$ & $d_{1}=2$ & $d_{1}=3$ & $d_{1}=4$ & $d_{1}=5$ \\
\hline \multirow{4}{*}{$\mu=0.3$} & {$[5]$} & 2.8119 & 2.8119 & 3.3173 & 4.0905 & - \\
& {$[13]$} & 3.0538 & 3.0129 & 3.3408 & 4.1690 & 5.0275 \\
& {$[14]$} & 3.1208 & 3.1092 & 3.4186 & 4.2097 & 5.0440 \\
& Corollary 3.3 & 3.1623 & 3.1754 & 3.4580 & 4.2576 & 5.0976 \\
\hline \multirow{4}{*}{$\mu=0.5$} & {$[5]$} & 2.3372 & 2.6181 & 3.3173 & 4.0905 & - \\
& {$[13]$} & 2.3058 & 2.5663 & 3.3408 & 4.1690 & 5.0275 \\
& {$[14]$} & 2.3513 & 2.6987 & 3.4186 & 4.2097 & 5.0440 \\
$\mu=0.9$ & Corollary 3.3 & 2.4594 & 2.7241 & 3.4580 & 4.2576 & 5.0976 \\
\hline \multirow{4}{*}{} & {$[5]$} & 2.0665 & 2.6181 & 3.3173 & 4.0905 & - \\
& {$[13]$} & 1.9008 & 2.5663 & 3.3408 & 4.1690 & 5.0275 \\
& {$[14]$} & 2.0921 & 2.6987 & 3.4186 & 4.2097 & 5.0440 \\
& Corollary 3.3 & 2.1207 & 2.7241 & 3.4580 & 4.2576 & 5.0976 \\
\hline
\end{tabular}

Example 4.2. Consider the time-delay system (3.30) with

$$
A=\left[\begin{array}{cc}
-2 & 0 \\
0 & -0.9
\end{array}\right], \quad B=\left[\begin{array}{cc}
-1 & 0 \\
-1 & -1
\end{array}\right]
$$

For various $\mu$, the allowable upper bounds $d_{2}$, which guarantee the stability of system (3.30) for given lower bounds $d_{1}$, are listed in Table 2. Moreover, the number of decision variables involved in the stability criteria are given in Table 3. From Tables 2 and 3, it can be seen that Corollary 3.3 in this paper has fewer decision variables and less conservatism than those results in $[5,13,14]$.

For unknown $\mu$, the allowable upper bounds $d_{2}$, which guarantee the stability of system (3.30) for given lower bounds $d_{1}$, are listed in Table 4. From Table 4, it can be seen that Corollary 3.6 in this paper give larger upper bounds of time delay than ones in $[4,5,8-$ $11,13,14]$.

Example 4.3. Consider the singular time-delay system (2.1) with

$$
E=\left[\begin{array}{ll}
1 & 0 \\
0 & 0
\end{array}\right], \quad A=\left[\begin{array}{cc}
-0.3012 & 0.1257 \\
0.2351 & -1.0998
\end{array}\right], \quad B=\left[\begin{array}{cc}
-0.5 c & 0 \\
0 & -0.1 c
\end{array}\right],
$$


Table 3: Number of decision variables.

\begin{tabular}{lc}
\hline Methods & Number of decision variables \\
\hline$[5]$ & $13 n^{2}+5 n$ \\
{$[13]$} & $18 n^{2}+8 n$ \\
{$[14]$} & $10.5 n^{2}+7.5 n$ \\
Corollary 3.3 & $9 n^{2}+5 n$ \\
\hline
\end{tabular}

Table 4: Allowable upper bound $d_{2}$ with given $d_{1}$ for unknown $\mu$.

\begin{tabular}{lllllc}
\hline Method & $d_{1}=1$ & $d_{1}=2$ & $d_{1}=3$ & $d_{1}=4$ & $d_{1}=5$ \\
\hline$[4]$ & 1.7424 & 2.4328 & 3.2234 & 4.0643 & - \\
{$[8]$} & 1.7661 & 2.4404 & 3.2260 & 4.0649 & - \\
{$[10]$} & 1.8737 & 2.5048 & 3.2591 & 4.0744 & - \\
{$[5,11]$} & 2.0665 & 2.6181 & 3.3173 & 4.0905 & - \\
{$[9]$} & 1.8043 & 2.5213 & 3.3311 & 4.1880 & 5.0722 \\
{$[13]$} & 1.9008 & 2.5663 & 3.3408 & 4.1690 & 5.0275 \\
{$[14]$} & 2.0921 & 2.6987 & 3.4186 & 4.2097 & 5.0440 \\
Corollary 3.6 & 2.1207 & 2.7241 & 3.4580 & 4.2576 & 5.0976 \\
\hline
\end{tabular}

Table 5: Allowable upper bound $d_{2}$ for different $c$.

\begin{tabular}{lccccc}
\hline$c$ & 1 & 1.2 & 1.4 & 1.6 & 2 \\
\hline$[18]$ & 2.0362 & 1.7691 & 1.5619 & 1.3977 & 1.1548 \\
{$[26](N=2)$} & 2.1660 & 1.8760 & 1.6470 & 1.4730 & 1.2160 \\
{$[22]$} & 2.2750 & 1.9635 & 1.7282 & 1.5438 & 1.2729 \\
{$[23,24]$} & 4.1762 & 3.1768 & 2.5740 & 2.1675 & 1.6509 \\
Corollary 3.8 & 4.4496 & 3.3657 & 2.7167 & 2.2814 & 1.7310 \\
\hline
\end{tabular}

where $c$ is a scalar. It is assumed that $d_{1}=0$ and $d(t)=d$ are constant delays satisfying $0 \leq d \leq d_{2}$. For various $c$, the allowable upper bounds $d_{2}$, which guarantee regular, impulse free, and stable of system (2.1), are listed in Table 5. From Table 5, it is clear that the Corollary 3.8 in this paper gives better results than those in $[18,22-24,26]$.

\section{Conclusion}

This paper deals with the problem of stability for singular systems with interval time-varying delay. A new stability criterion for singular systems to be regular, impulse free, and stable is proposed in terms of LMIs. Based on the obtained criterion, some improved stability results for the regular systems with interval time-varying delay are also given. The obtained results in this paper have been shown to be less conservative than recently reported results. Moreover, the proposed method decreases the computational complexity comparable to some existing methods. Three numerical examples are given to illustrate the applicability of the results. 


\section{Acknowledgment}

The author is grateful to the academic editor and four anonymous referees whose comments have helped to improve the final version of the present work. This work was supported by the scientific research program funded by Shaanxi provincial education department (nos. 2010JK400 and 11JK0509) and the scientific research project funded by Baoji University of Arts and Sciences (no. 11044).

\section{References}

[1] K. Gu, V. Kharitonov, and J. Chen, Stability of Time-Delay Systems, Birkhauser, Boston, Mass, USA, 2003.

[2] M. Wu, Y. He, and J.-H. She, Stability Analysis and Robust Control of Time-Delay Systems, Science Press, Beijing, China, 2010.

[3] Q. L. Han and K. Gu, "Stability of linear systems with time-varying delay: a generalized discretized lyapunov functional approach," Asian Journal of Control, vol. 3, no. 3, pp. 170-180, 2001.

[4] Y. He, Q.-G. Wang, C. Lin, and M. Wu, "Delay-range-dependent stability for systems with timevarying delay," Automatica, vol. 43, no. 2, pp. 371-376, 2007.

[5] W. Zhang, X.-S. Cai, and Z.-Z. Han, "Robust stability criteria for systems with interval time-varying delay and nonlinear perturbations," Journal of Computational and Applied Mathematics, vol. 234, no. 1 pp. 174-180, 2010.

[6] T. Li, L. Guo, and Y. Zhang, "Delay-range-dependent robust stability and stabilization for uncertain systems with time-varying delay," International Journal of Robust and Nonlinear Control, vol. 18, no. 13, pp. 1372-1387, 2008.

[7] O. M. Kwon and J. H. Park, “Delay-range-dependent stabilization of uncertain dynamic systems with interval time-varying delays," Applied Mathematics and Computation, vol. 208, no. 1, pp. 58-68, 2009.

[8] H. Shao, "Improved delay-dependent stability criteria for systems with a delay varying in a range," Automatica, vol. 44, no. 12, pp. 3215-3218, 2008.

[9] X. Jiang and Q.-L. Han, "New stability criteria for linear systems with interval time-varying delay," Automatica, vol. 44, no. 10, pp. 2680-2685, 2008.

[10] H. Shao, "New delay-dependent stability criteria for systems with interval delay," Automatica, vol. 45, no. 3, pp. 744-749, 2009.

[11] P. Park, J. W. Ko, and C. Jeong, "Reciprocally convex approach to stability of systems with timevarying delays," Automatica, vol. 47, no. 1, pp. 235-238, 2011.

[12] J. Sun, G. P. Liu, J. Chen, and D. Rees, "Improved stability criteria for linear systems with time-varying delay," IET Control Theory \& Applications, vol. 4, no. 4, pp. 683-689, 2010.

[13] J. Sun, G. P. Liu, J. Chen, and D. Rees, "Improved delay-range-dependent stability criteria for linear systems with time-varying delays," Automatica, vol. 46, no. 2, pp. 466-470, 2010.

[14] Y. Liu, L.-S. Hu, and P. Shi, "A novel approach on stabilization for linear systems with time-varying input delay," Applied Mathematics and Computation, vol. 218, no. 10, pp. 5937-5947, 2012.

[15] J. K. Hale and S. M. Verduyn Lunel, Introduction to Functional-Differential Equations, vol. 99, Springer, New York, NY, USA, 1993.

[16] L. Dai, Singular Control Systems, vol. 118, Springer, Berlin, Germany, 1989.

[17] V. B. Kolmanovskiř and V. R. Nosov, Stability of Functional-Differential Equations, vol. 180, Academic Press, New York, NY, USA, 1986.

[18] E. Fridman and U. Shaked, " $H_{\infty}$-control of linear state-delay descriptor systems: an LMI approach," Linear Algebra and its Applications, vol. 351-352, pp. 271-302, 2002.

[19] R. Zhong and Z. Yang, "Delay-dependent robust control of descriptor systems with time delay," Asian Journal of Control, vol. 8, no. 1, pp. 36-44, 2006.

[20] H. Su, X. Ji, and J. Chu, "Delay-dependent robust control for uncertain singular time-delay systems," Asian Journal of Control, vol. 8, no. 2, pp. 180-189, 2006.

[21] Z. Wu and W. Zhou, "Delay-dependent robust $H_{\infty}$ control for uncertain singular time-delay systems," IET Control Theory \& Applications, vol. 1, no. 5, pp. 1234-1241, 2007.

[22] S. Xu, J. Lam, and Y. Zou, "An improved characterization of bounded realness for singular delay systems and its applications," International Journal of Robust and Nonlinear Control, vol. 18, no. 3, pp. 263-277, 2008. 
[23] Z.-G. Wu, H.-Y. Su, and J. Chu, "Improved results on delay-dependent $H_{\infty}$ control for singular timedelay systems," Acta Automatica Sinica, vol. 35, no. 8, pp. 1101-1106, 2009.

[24] X. Sun, Q. L. Zhang, C. Y. Yang, Z. Su, and Y. Y. Shao, "An improved approach to delay-dependent robust stabilization for uncertain singular time-delay systems," International Journal of Automation and Computing, vol. 7, no. 2, pp. 205-212, 2010.

[25] Y.-F. Feng, X.-L. Zhu, and Q.-L. Zhang, "Delay-dependent stability criteria for singular time-delay systems," Acta Automatica Sinica, vol. 36, no. 3, pp. 433-437, 2010.

[26] L.-L. Liu, J.-G. Peng, and B.-W. Wu, “On parameterized Lyapunov-Krasovskii functional techniques for investigating singular time-delay systems," Applied Mathematics Letters, vol. 24, no. 5, pp. 703-708, 2011.

[27] S. M. Saadni, M. Chaabane, and D. Mehdi, "Robust stability and stabilization of a class of singular systems with multiple time-varying delays," Asian Journal of Control, vol. 8, no. 1, pp. 1-11, 2006.

[28] E.-K. Boukas, "Delay-dependent robust stabilizability of singular linear systems with delays," Stochastic Analysis and Applications, vol. 27, no. 4, pp. 637-655, 2009.

[29] Z. Wu, H. Su, and J. Chu, "Robust stabilization for uncertain discrete singular systems with state delay," International Journal of Robust and Nonlinear Control, vol. 18, no. 16, pp. 1532-1550, 2008.

[30] J.-M. Jiao, "Robust stability and stabilization of discrete singular systems with interval time-varying delay and linear fractional uncertainty," Internal Journal of Automation and Computing, vol. 9, no. 1, pp. 8-15, 2012.

[31] D. Zhang, L. Yu, Q.-G. Wang, C.-J. Ong, and Z.-G. Wu, “Exponential $H_{\infty}$ filtering for discrete-time switched singular systems with time-varying delays," Journal of Franklin Institute, vol. 349, no. 7, pp. 2323-2342, 2012.

[32] Z.-G. Feng and J. Lam, "Robust reliable dissipative filtering for discrete delay singular systems," Signal Processing, vol. 92, no. 12, pp. 3010-3025, 2012.

[33] Z. Wu, H. Su, and J. Chu, " $H_{\infty}$ filtering for singular systems with time-varying delay," International Journal of Robust and Nonlinear Control, vol. 20, no. 11, pp. 1269-1284, 2010.

[34] Z. Wu, H. Su, and J. Chu, "Delay-dependent $H_{\infty}$ filtering for singular Markovian jump time-delay systems," Signal Processing, vol. 90, no. 6, pp. 1815-1824, 2010.

[35] J. Lin and S. Fei, "Reliable control for a class of uncertain singular systems with interval time-varying delay," Asian Journal of Control, vol. 13, no. 4, pp. 542-552, 2011.

[36] Y. Ding, S. Zhong, and W. Chen, "A delay-range-dependent uniformly asymptotic stability criterion for a class of nonlinear singular systems," Nonlinear Analysis. Real World Applications, vol. 12, no. 2, pp. 1152-1162, 2011.

[37] Z.-G. Wu, J. H. Park, H. Su, and J. Chu, "Dissipativity analysis for singular systems with time-varying delays," Applied Mathematics and Computation, vol. 218, no. 8, pp. 4605-4613, 2011.

[38] P. Balasubramaniam, R. Krishnasamy, and R. Rakkiyappan, “Delay-dependent stability criterion for a class of non-linear singular Markovian jump systems with mode-dependent interval time-varying delays," Communications in Nonlinear Science and Numerical Simulation, vol. 17, no. 9, pp. 3612-3627, 2012.

[39] P. Balasubramaniam, R. Krishnasamy, and R. Rakkiyappan, “Delay-dependent stability of neutral systems with time-varying delays using delay-decomposition approach," Applied Mathematical Modelling, vol. 36, no. 5, pp. 2253-2261, 2012. 


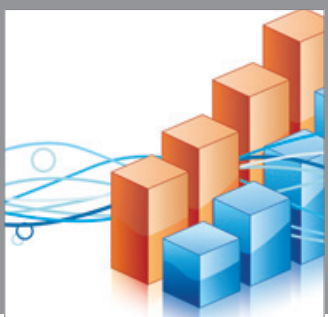

Advances in

Operations Research

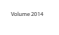

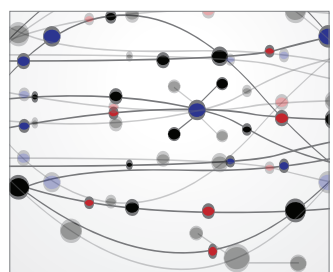

\section{The Scientific} World Journal
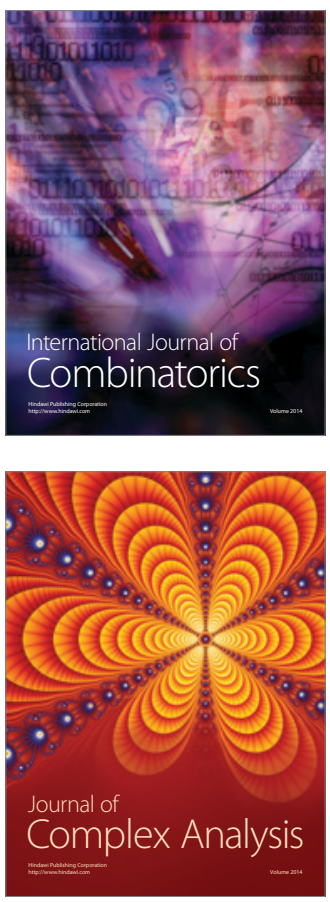

International Journal of

Mathematics and

Mathematical

Sciences
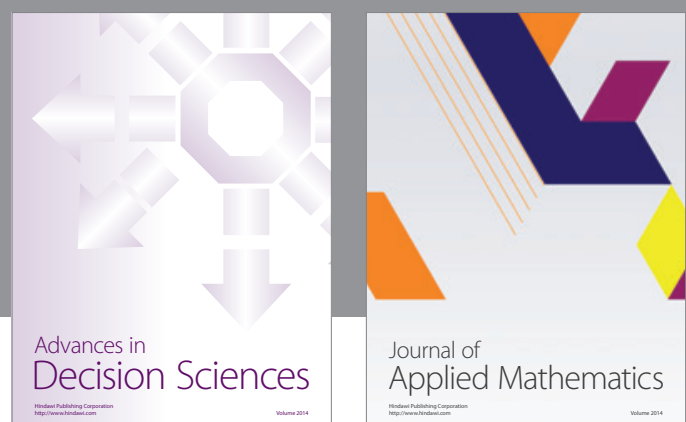

Journal of

Applied Mathematics
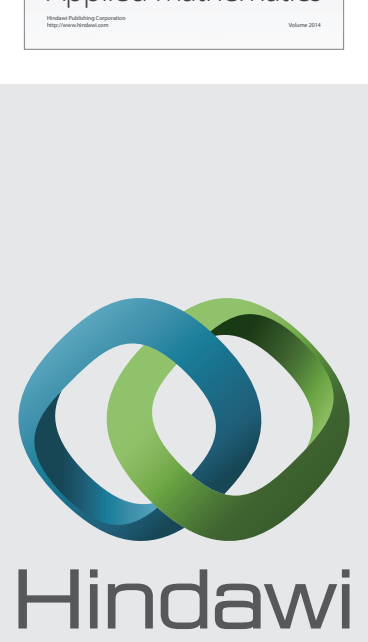

Submit your manuscripts at http://www.hindawi.com
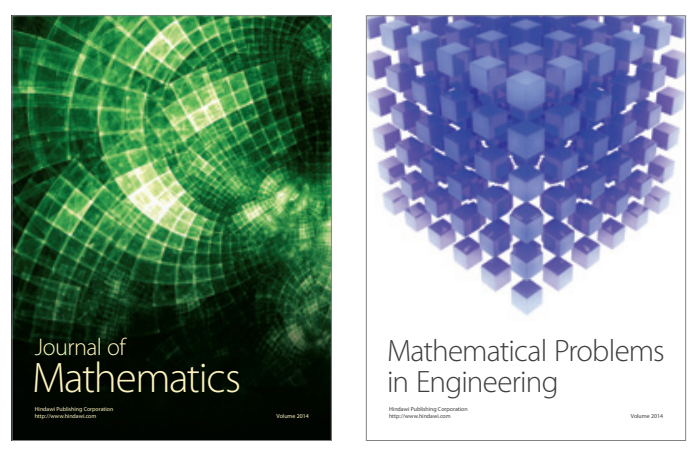

Mathematical Problems in Engineering
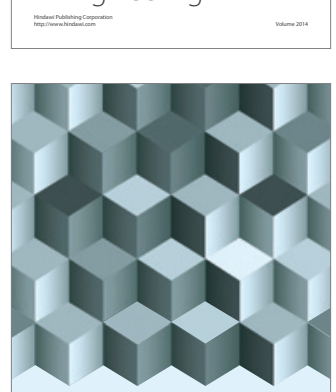

Journal of

Function Spaces
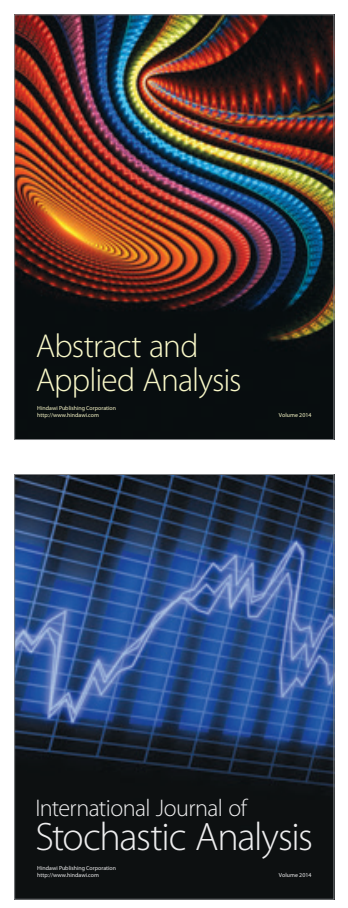

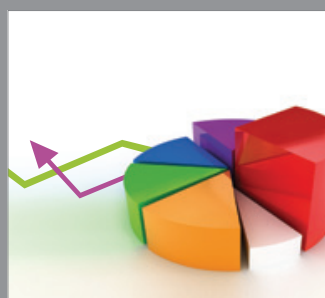

ournal of

Probability and Statistics

Promensencen
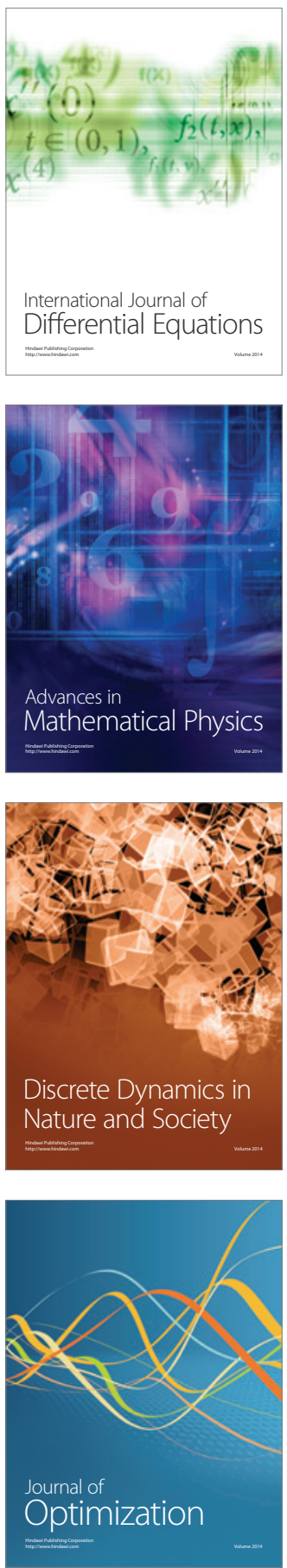